\title{
Nível de resposta das emissões otoacústicas evocadas por estímulo transiente em lactentes com refluxo gastroesofágico
}

\section{Response level of the transient-evoked otoacoustic emissions on infants having a gastroesophageal reflux}

\author{
Elizângela Dias Camboim 1 , Renata Coelho Scharlach², Kelvânio Vitório de Farias ${ }^{3}$, Lívia Karla Gadêlha de Oliveira4, \\ Dênis Vasconcelos ${ }^{5}$, Marisa Frasson de Azevedo ${ }^{6}$.
}
1) Mestranda em distúrbios da comunicação humana pela Universidade Federal de São Paulo (UNIFESP). Professora Auxiliar da Faculdade de Fonoaudiologia de Alagoas da Universidade Estadual de Ciências da Saúde de Alagoas (UNCISAL).
2) Doutora em Distúrbios da Comunicação Humana pela Universidade Federal de São Paulo (UNIFESP). Docente colaboradora da Disciplina de Distúrbios da Audição do Departamento de Fonoaudiologia da Universidade Federal de São Paulo (UNIFESP).
3) Especialização em Audiologia pela Universidade Estadual de Ciências da Saúde de Alagoas (UNCISAL). Residente em audiologia pela Universidade Estadual de Ciências da Saúde de Alagoas (UNCISAL)
4) Especialização em Audiologia Clínica pelo Instituto Materno Infantil de Pernambuco. Residente em audiologia pela Universidade Estadual de Ciências da saúde de Alagoas (UNCISAL).
5) Otorrinolaringologista. Médico do Trabalho e do Trânsito
6) Doutora em Distúrbios da Comunicação Humana pela Universidade Federal de São Paulo (UNIFESP). Professor Associado Doutor da Universidade Federal de São Paulo (UNIFESP).

Instituição: Universidade Estadual de Ciências da Saúde de Alagoas (UNCISAL).

Arapiraca / AL - Brasil.

Endereço para correspondência: Kelvânio Vitório de Farias - Travessa Manoel Afonso Maranhão, 83 - Jardim Esperança - Arapiraca / Al - Brasil - CEP: $57307-300$ - Telefone: (+55 82) 9932-0729 - E-mail: k_fono@hotmail.com

Artigo recebido em 19 de Janeiro de 2011. Artigo aprovado em 19 de Abril de 2011.

\section{RESUMO}

Introdução: As emissões otoacústicas evocadas por estímulo transiente (EOAT) têm sido a técnica mais utilizada para a realização da triagem auditiva neonatal. Sendo importante analisar suas medidas correlacionando com outras alterações que pode acometer o sistema auditivo da criança.

Objetivo: Analisar a ocorrência e os níveis de resposta das emissões otoacústicas evocadas por estímulo transiente em lactentes com refluxo gastroesofágico fisiológico (RGEF).

Método: Estudo prospectivo foi realizado no Serviço de Otorrinolaringologia do Hospital Santa Juliana. Participaram do estudo 118 bebês, de recém nascidos há seis meses, encaminhados por pediatras e gastropediatras, nascidos pré-temo ou termo e distribuídos em dois grupos: Grupo Estudo: 63 lactentes com diagnóstico clínico de refluxo gastroesofágico fisiológico, e Grupo Controle: 55 lactentes sem refluxo gastroesofágico fisiológico. Foi realizada a avaliação da função auditiva periférica por meio dos exames de emissões otoacústicas evocadas por estímulo transiente e otoscopia realizada por otorrinolaringologista.

Resultados: Os níveis médios de respostas das emissões otoacústicas evocadas por estímulo transiente foram maiores no grupo sem refluxo para as bandas de frequências de $2 \mathrm{kHz}$, $2,5 \mathrm{kHz}, 3 \mathrm{kHz}, 3,5 \mathrm{kHz}$ e $4,5 \mathrm{kHz}$ bilateralmente, com diferenças estatisticamente significantes, obtendo-se valores médios de $7,71 \mathrm{~dB}$ e $7 \mathrm{~dB}$ na orelha direita, encontrados nas bandas de frequências de 2 e $4 \mathrm{kHz}$, respectivamente.

Conclusão: Houve menor ocorrência e menor nível de respostas das emissões otoacústicas transientes em crianças com refluxo gastroesofágico fisiológico quando comparados a crianças sem refluxo.

Palavras-chave: refluxo gastroesofágico, emissões otoacústicas espontâneas, audição, lactente.

\section{SUMMARY}

Introduction: The transient-evoked otoacoustic emissions (TEOAE) have been the most widespread technique to perform neonatal hearing screening. Scrutinizing their measures by way of an association with other alterations that may impair the infant's auditory system is important.

Objective: Analyze the incidence and the response levels of the transient-evoked otoacoustic emissions on infants having a physiological gastroesophageal reflux disease (GERD).

Method: A prospective study was performed at Santa Juliana Hospital's Otorhinolaryngology Department. 118 prematurelyborn and timely-born babies, from newly-born to 6 months old, who were sent by pediatricians and gastropediatricians, participated in the study and they were divided into two groups: Study Group: 63 infants clinically diagnosed of a physiological gastroesophageal reflux disease, and Control Group: 55 infants without a physiological gastroesophageal reflux. The peripheral hearing function was evaluated by both transient-evoked otoacoustic emissions and otoscopy examinations performed by an otorhinolaryngologist.

Results: The average response levels of the transient-evoked otoacoustic emissions were higher in the non-reflux group for frequency bands of $2 \mathrm{kHz}, 2.5 \mathrm{kHz}, 3 \mathrm{kHz}, 3.5 \mathrm{kHz}$ and $4.5 \mathrm{kHz}$ bilaterally, with a statistically significant difference, achieving the average values of $7.71 \mathrm{~dB}$ and $7 \mathrm{~dB}$ in the right ear found in the frequency bands of 2 and $4 \mathrm{kHz}$, respectively.

Conclusion: There was a lower incidence and a lower response level of the transient-evoked otoacoustic emissions in physiological gastroesophageal reflux children in comparison with children having no reflux.

Keywords: gastroesophageal reflux, spontaneous otoacoustic emissions, hearing, infant. 


\section{INTRODUÇÃO}

As Emissões Otoacústicas (EOA) são sons subliminares que emergem do meato acústico externo, quando a membrana timpânica recebe vibrações vindas da cóclea, transmitidas pela orelha média (1). Essas vibrações ocorrem como um subproduto de um mecanismo coclear específico e vulnerável que se tornou conhecido como amplificador coclear e que contribui significativamente para a sensibilidade auditiva e a discriminação de frequências (2).

Na prática clínica as EOA podem ser registradas de maneira espontânea ou evocadas acusticamente por meio de estímulo transiente ou produto de distorção (3).

As EOA evocadas são registradas na maioria dos indivíduos que apresentam função coclear normal, independente da idade e sexo. Sua presença indica a integridade das células ciliadas externas da cóclea (4).

Para a captação das EOA é necessária a integridade da orelha externa e média, pois qualquer alteração na transmissão do estímulo acústico poderá acarretar diminuição ou ausência de resposta, comprometendo a análise do exame (5).

Na pratica clínica é possível observar uma relação entre a diminuição dos níveis de resposta das emissões ou até mesmo ausência de resposta em pacientes que apresentam alterações de orelha media (6).

As causas mais comuns das alterações de orelha média na criança estão relacionadas à perfuração do tímpano, infecção ou líquido na orelha média e mais recentemente foi incluído o refluxo gastroesofágico, o qual pode contribuir significativamente para a inflamação de orelha média e participar da fisiopatologia das otites com efusão, por ação do ácido pepsina e pepsinogênio $(7,8)$.

O refluxo gastroesofágico (RGE) é mais comum nos primeiros meses de vida. As regurgitações pós-alimentares surgem entre o nascimento e os quatro meses de idade, apresentando resolução espontânea, na maioria dos casos, até um a dois anos de idade $(9,10)$.

O RGE foi classificado como fisiológico em lactentes entre um e 12 meses, quando apresentavam dois ou mais episódios de regurgitação ao dia por período superior a três semanas, sem história de hematêmese, broncoaspiração, apneia, déficit pondero-estatural ou postura anormal (11).

A regurgitação tende a desaparecer naturalmente até 12 meses, no entanto não significa que o refluxo não esta mais presente, pois em aproximadamente $5 \%$ dos casos a permanência do refluir sem regurgitação, pode causar sintomas otorrinolaringológico como: laringites, sinusites e otites (12).

Tendo em vista a quantidade resumida de trabalhos correlacionando as emissões otoacústicas evocadas em bebês com RGE e mediante a necessidade desta avaliação na triagem auditiva neonatal (TAN), o objetivo deste trabalho foi analisar a ocorrência e os níveis de resposta das emissões otoacústicas evocadas por estímulo transiente em lactentes com RGE fisiológico.

\section{MÉTOdo}

A coleta iniciou após a aprovação do Comitê de Ética em Pesquisa da Universidade Estadual de Ciências da Saúde de Alagoas UNCISAL, protocolo oㅜ 583. Estudo do tipo prospectivo realizado no Serviço de Otorrinolaringologia do Hospital Santa Juliana, Maceió Alagoas. (Instituição Filantrópica Municipal) e segue a Resolução 196/96 do conselho nacional de saúde. O termo de consentimento livre e esclarecido foi lido e explicado aos responsáveis pelos bebês, os quais concordaram e assinaram.

A amostra foi constituída por 118 lactentes, de ambos os sexos, com faixa etária de recém nascido há 6 meses, nascidos pré temo ou termo, distribuídos em dois grupos: Grupo estudo (GE), formado por 63 lactentes com refluxo, com diagnóstico clínico de médicos pediatras ou gastropediatras. Esse diagnostico foi realizado por meio de um formulário baseado no critério de ROMA II que relata os sintomas do RGE. Quando identificado pelo menos 2 sintomas o bebê foi incluído no grupo com refluxo gastroesofágico fisiológico. Não foi identificado lactentes com refluxo patológico. O grupo controle (GC) formado por 55 lactentes sem refluxo, pareados por idade, sexo e idade gestacional.

O grupo estudo, com refluxo foi composto por 36 crianças do gênero feminino e 27 do masculino, e o grupo sem refluxo por 30 crianças do gênero feminino e 25 do masculino. E em relação à idade gestacional (nascidos a termo e pré-termo), o grupo com refluxo foi formado por 18 crianças pré-termo e 45 a termo; no grupo sem refluxo, 16 crianças eram pré-termo e 39 a termo.

Foram excluídos lactentes com fissura lábio palatina ou má formação de orelha externa e ou média, má formação de cabeça e pescoço, síndromes genéticas associadas a alterações auditivas, crianças com história familiar de perda auditiva, alterações neurológicas e Displasia bronco-pulmonar. 
O cálculo do tamanho da amostra foi realizado no programa Statcalt no Epi info, versão 6.04. Onde foi atribuído um erro alfa e beta de 5\%. Foi obtido um valor de amostra mínima de 82 lactentes.

Para analisar o RGE em relação à análise das amplitude e sinal/ruído das EOAT foi utilizado o Teste de MannWhitney e para a associação do uso de medicação na ocorrência das EOAT foi utilizado o teste Qui-Quadrado para Independência.

Foi realizada avaliação otorrinolaringológica em todos os sujeitos da pesquisa, por meio da otoscopia com intuito de verificar integridade orelha externa e média

As emissões otoacústicas evocadas por estímulo transiente (EOAT) foram realizadas pela pesquisadora responsável numa clínica otorrinolaringológica em ambiente com baixo nível de ruído e com o lactente em estado de sono natural no colo da mãe. Os exames foram realizados com equipamento otoRead da marca interacoustic, que permite a captação das respostas das emissões otoacústicas, por meio da colocação de uma sonda (com microfone acoplado) no meato acústico externo.

Utilizou-se como critério de análise, o parâmetro de PASSA /FALHA descritos no protocolo do próprio equipamento com estímulo: clique; intensidade: $83 \mathrm{dBpeSPL}$; número de bandas de frequências testadas: 6 (de $1500 \mathrm{~Hz}$ a $4000 \mathrm{~Hz}$ ). Os valores para considerar teste PASSA foram: Emissões presentes numa relação sinal/ruído de 4 dB em pelo menos três bandas de frequência. O tempo de captação das EOA no equipamento é de no máximo, 64 segundos, podendo parar automaticamente antes do tempo quando o resultado for considerado presente.

As duas avaliações (otoscopia e EOAT) foram realizadas separadamente, porém no mesmo dia, sem que o examinadores tivessem conhecimento dos resultados desta avaliações, antes da finalização de todos os teste.

\section{RESULTADOS}

Os resultados serão a presentados comparando-se os grupos com e sem RGE na distribuição de frequência relativa (percentuais) das variáveis qualitativas.

A ocorrência de EOAT nos grupos estudados a parece descrita nos Gráficos 1 e 2.

Ao se comparar os resultados da ocorrência das EOAT entre os grupos, observou-se maior prevalência de EOAT presentes no grupo sem refluxo. Contudo, foi possível também observar que, mesmo no grupo com

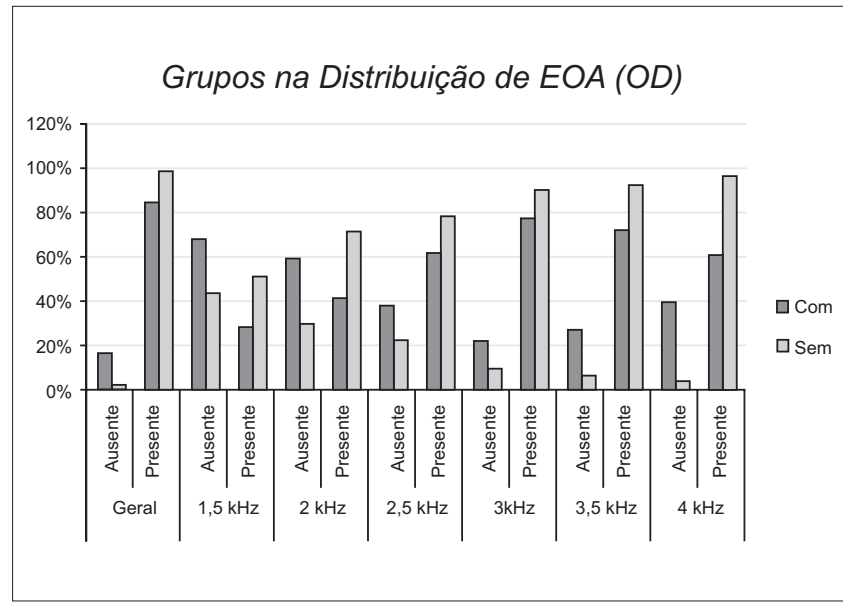

Gráfíco1. Comparação dos grupos na distribuição da ocorrência das EOA na OD.

Legenda: OD: orelha direita; EOA: emissões otoacústicas; com: grupo com refluxo; sem: grupo sem refluxo.

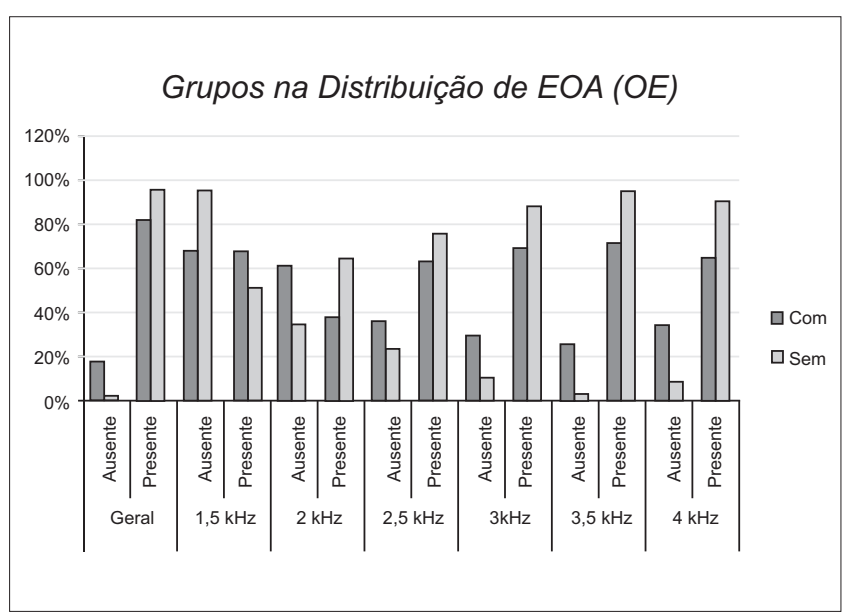

Gráfico 2. Comparação dos grupos na distribuição da ocorrência das EOA na OE.

Legenda: OE: orelha esquerda; EOA: emissões otoacústicas; com: grupo com refluxo; sem: grupo sem refluxo.

refluxo, houve uma maior ocorrência de EOAT presentes quando comparado aos resultados ausentes.

Na análise da ocorrência do uso de medicamento no grupo com refluxo observou-se que $81 \%$ do grupo com RGE nunca fizeram uso de medicamentos.

Em relação à associação da medicação com a ocorrência das EOAT foram observados os resultados descritos na Tabela 1. O teste estatístico utilizado para esta análise foi o Qui-Quadrado.

Verificou-se que houve associação estatisticamente significante entre medicamentos e o resultado das EOAT 
Tabela I. Associação do uso de medicamentos e ocorrência das EOAT em ambas as orelhas.

\begin{tabular}{|c|c|c|c|c|c|c|c|c|c|c|}
\hline \multicolumn{2}{|l|}{ EOAT } & \multicolumn{2}{|c|}{ EmUso } & \multicolumn{2}{|c|}{ JáUsou } & \multicolumn{2}{|c|}{ Não Usou } & \multicolumn{2}{|c|}{ Total } & \multirow[t]{2}{*}{ p-valor } \\
\hline & & $\mathrm{N}$ & $\%$ & $\mathrm{~N}$ & $\%$ & $\mathrm{~N}$ & $\%$ & $\mathrm{~N}$ & $\%$ & \\
\hline \multirow[t]{3}{*}{ OD } & Falha & 3 & $33 \%$ & 0 & $0 \%$ & 8 & $8 \%$ & 11 & $9 \%$ & \multirow[t]{3}{*}{$0,029 *$} \\
\hline & Passa & 6 & $67 \%$ & 6 & $100 \%$ & 95 & $92 \%$ & 107 & $91 \%$ & \\
\hline & TOTAL & 9 & $100 \%$ & 6 & $100 \%$ & 103 & $100 \%$ & 118 & $100 \%$ & \\
\hline \multirow[t]{3}{*}{ OE } & Falha & 3 & $33 \%$ & I & $17 \%$ & 9 & $9 \%$ & 13 & $11 \%$ & \multirow[t]{3}{*}{0,07} \\
\hline & Passa & 6 & $67 \%$ & 5 & $83 \%$ & 94 & $91 \%$ & 105 & $89 \%$ & \\
\hline & TOTAL & 9 & $100 \%$ & 6 & $100 \%$ & 103 & $100 \%$ & 118 & $100 \%$ & \\
\hline \multirow[t]{3}{*}{ Ambas } & Falha & 6 & $33 \%$ & I & $8 \%$ & 17 & $8 \%$ & 24 & $10 \%$ & \multirow[t]{3}{*}{$0,003 *$} \\
\hline & Passa & 12 & $67 \%$ & | | & $92 \%$ & 189 & $92 \%$ & 212 & $90 \%$ & \\
\hline & TOTAL & 18 & $100 \%$ & 12 & $100 \%$ & 206 & $100 \%$ & 236 & $100 \%$ & \\
\hline
\end{tabular}

Legenda: *p-valor< 0,05. Legenda: EOAT: emissões otoacústicas por estímulo transiente; OD: orelha direita; OE: orelha esquerda.

Tabela 2. Comparação da média da relação sinal ruído das EOAT entre os grupos estudados.

\begin{tabular}{|c|c|c|c|c|c|c|c|c|c|}
\hline \multicolumn{2}{|c|}{ EOATS/R } & Média & Mediana & Desvio Padrão & QI & Q3 & $\mathrm{N}$ & IC & p-valor \\
\hline $\mathrm{OD}(\mathrm{I}, 5 \mathrm{kHz})$ & $\begin{array}{l}\text { ComRGE } \\
\text { SemRGE }\end{array}$ & $\begin{array}{l}1,76 \\
5,33\end{array}$ & $\begin{array}{l}1,0 \\
5,0\end{array}$ & $\begin{array}{l}5,49 \\
6,23\end{array}$ & $\begin{array}{l}-2,0 \\
0,0\end{array}$ & $\begin{array}{l}5,0 \\
10,0\end{array}$ & $\begin{array}{l}63 \\
55\end{array}$ & $\begin{array}{l}1,36 \\
1,65\end{array}$ & 0,00 I* \\
\hline $\mathrm{OD}(2 \mathrm{kHz})$ & $\begin{array}{l}\text { ComRGE } \\
\text { SemRGE }\end{array}$ & $\begin{array}{l}3,17 \\
7,82\end{array}$ & $\begin{array}{l}2,0 \\
7,0\end{array}$ & $\begin{array}{l}5,71 \\
6,54\end{array}$ & $\begin{array}{r}-0,5 \\
4,0\end{array}$ & $\begin{array}{l}6,0 \\
11,0\end{array}$ & $\begin{array}{l}63 \\
55\end{array}$ & $\begin{array}{l}1,4 \mid \\
1,73\end{array}$ & $<0,00$ |* \\
\hline $\mathrm{OD}(2,5 \mathrm{kHz})$ & $\begin{array}{l}\text { ComRGE } \\
\text { SemRGE }\end{array}$ & $\begin{array}{c}6,03 \\
10,73\end{array}$ & $\begin{array}{l}5,0 \\
11,0\end{array}$ & $\begin{array}{l}7,07 \\
6,20\end{array}$ & $\begin{array}{l}1,5 \\
6,5\end{array}$ & $\begin{array}{l}9,0 \\
15,0\end{array}$ & $\begin{array}{l}63 \\
55\end{array}$ & $\begin{array}{l}1,75 \\
1,64\end{array}$ & $<0,00$ |* \\
\hline $\mathrm{OD}(3 \mathrm{kHz})$ & $\begin{array}{l}\text { ComRGE } \\
\text { SemRGE }\end{array}$ & $\begin{array}{l}7,97 \\
11,96\end{array}$ & $\begin{array}{l}7,0 \\
11,0\end{array}$ & $\begin{array}{l}6,21 \\
6,57\end{array}$ & $\begin{array}{l}4,5 \\
7,0\end{array}$ & $\begin{array}{l}11,5 \\
16,0\end{array}$ & $\begin{array}{l}63 \\
55\end{array}$ & $\begin{array}{l}1,53 \\
1,74\end{array}$ & 0,00 I* \\
\hline $\mathrm{OD}(3,5 \mathrm{kHz})$ & $\begin{array}{l}\text { ComRGE } \\
\text { SemRGE }\end{array}$ & $\begin{array}{l}8,79 \\
13,13\end{array}$ & $\begin{array}{l}8,0 \\
13,0\end{array}$ & $\begin{array}{l}6,89 \\
7,09\end{array}$ & $\begin{array}{l}4,5 \\
8,5\end{array}$ & $\begin{array}{l}14,0 \\
18,0\end{array}$ & $\begin{array}{l}63 \\
55\end{array}$ & $\begin{array}{l}1,70 \\
1,88\end{array}$ & $0,00 \mid *$ \\
\hline $\mathrm{OD}(4 \mathrm{kHz})$ & $\begin{array}{l}\text { ComRGE } \\
\text { SemRGE }\end{array}$ & $\begin{array}{l}6,75 \\
14,55\end{array}$ & $\begin{array}{l}7,0 \\
13,0\end{array}$ & $\begin{array}{l}6,87 \\
7,89\end{array}$ & $\begin{array}{l}1,0 \\
10,0\end{array}$ & $\begin{array}{l}11,0 \\
18,0\end{array}$ & $\begin{array}{l}63 \\
55\end{array}$ & $\begin{array}{l}1,70 \\
2,08\end{array}$ & $<0,00$ |* \\
\hline $\mathrm{OE}(\mathrm{I}, 5 \mathrm{kHz})$ & $\begin{array}{l}\text { ComRGE } \\
\text { SemRGE }\end{array}$ & $\begin{array}{l}1,68 \\
3,93\end{array}$ & $\begin{array}{l}1,0 \\
4,0\end{array}$ & $\begin{array}{l}5,11 \\
6,35\end{array}$ & $\begin{array}{r}-2,0 \\
0,0\end{array}$ & $\begin{array}{l}4,5 \\
9,5\end{array}$ & $\begin{array}{l}63 \\
55\end{array}$ & $\begin{array}{l}1,26 \\
1,68\end{array}$ & $0,032 *$ \\
\hline $\mathrm{OE}(2 \mathrm{kHz})$ & $\begin{array}{l}\text { ComRGE } \\
\text { SemRGE }\end{array}$ & $\begin{array}{l}3,59 \\
6,85\end{array}$ & $\begin{array}{l}3,0 \\
7,0\end{array}$ & $\begin{array}{l}6,92 \\
5,33\end{array}$ & $\begin{array}{l}-2,0 \\
2,5\end{array}$ & $\begin{array}{l}7,5 \\
10,5\end{array}$ & $\begin{array}{l}63 \\
55\end{array}$ & $\begin{array}{l}|, 7| \\
|, 4|\end{array}$ & $0,002 *$ \\
\hline $\mathrm{OE}(2,5 \mathrm{kHz})$ & $\begin{array}{l}\text { ComRGE } \\
\text { SemRGE }\end{array}$ & $\begin{array}{l}4,71 \\
9,31\end{array}$ & $\begin{array}{l}6,0 \\
9,0\end{array}$ & $\begin{array}{l}10,13 \\
6,56\end{array}$ & $\begin{array}{l}1,0 \\
5,0\end{array}$ & $\begin{array}{l}10,0 \\
14,0\end{array}$ & $\begin{array}{l}63 \\
55\end{array}$ & $\begin{array}{l}2,50 \\
1,73\end{array}$ & $0,003 *$ \\
\hline $\mathrm{OE}(3 \mathrm{kHz})$ & $\begin{array}{l}\text { ComRGE } \\
\text { SemRGE }\end{array}$ & $\begin{array}{l}6,62 \\
|1,5|\end{array}$ & $\begin{array}{l}6,0 \\
11,0\end{array}$ & $\begin{array}{l}7,23 \\
6,45\end{array}$ & $\begin{array}{l}4,0 \\
7,0\end{array}$ & $\begin{array}{l}9,5 \\
17,0\end{array}$ & $\begin{array}{l}63 \\
55\end{array}$ & $\begin{array}{l}1,78 \\
1,7 \mid\end{array}$ & $<0,00 \mid *$ \\
\hline $\mathrm{OE}(3,5 \mathrm{kHz})$ & $\begin{array}{l}\text { ComRGE } \\
\text { SemRGE }\end{array}$ & $\begin{array}{l}6,84 \\
12,44\end{array}$ & $\begin{array}{l}8,0 \\
12,0\end{array}$ & $\begin{array}{l}8,98 \\
6,74\end{array}$ & $\begin{array}{l}4,0 \\
7,0\end{array}$ & $\begin{array}{l}12,0 \\
16,0\end{array}$ & $\begin{array}{l}63 \\
55\end{array}$ & $\begin{array}{l}2,22 \\
1,78\end{array}$ & $<0,00 \mid *$ \\
\hline $\mathrm{OE}(4 \mathrm{kHz})$ & $\begin{array}{l}\text { ComRGE } \\
\text { SemRGE }\end{array}$ & $\begin{array}{l}5,59 \\
13,02\end{array}$ & $\begin{array}{l}6,0 \\
11,0\end{array}$ & $\begin{array}{l}6,94 \\
8,55\end{array}$ & $\begin{array}{l}0,0 \\
7,0\end{array}$ & $\begin{array}{l}9,5 \\
19,5\end{array}$ & $\begin{array}{l}63 \\
55\end{array}$ & $\begin{array}{l}1,71 \\
2,26\end{array}$ & $<0,00$ |* \\
\hline
\end{tabular}

Legenda: *p-valor:<0,05; EOAT: emissões otoacústicas por estímulo transiente; OD: orelha direita; OE: orelha esquerda.

em ambas as orelhas, como também na Orelha Direita. Houve diferença estatisticamente significante nos lactentes que usaram medicação em relação aos que não usaram. Os lactentes que estão em uso da medicação apresentaram maior ocorrência percentual de falha nas EOAT, quando comparados aos demais percentuais de falha dos lactentes que já fizeram uso ou que nunca usaram a medicação.
Para avaliar a relação sinal ruído das EOAT em ambos os grupos foi utilizado o teste de Mann-Whitney sendo observados os resultados na Tabela 2.

Observou-se diferença estatisticamente significante entre os grupos para todas as bandas de frequências em ambas as orelhas. Ou seja, no grupo controle, grupo sem 
Tabela 3. Medidas descritivas e comparação do nível médio de resposta das EOAT obtidas nas diferentes bandas de frequência estudadas para o Grupo com refluxo e para o Grupo Sem refluxo.

\begin{tabular}{|c|c|c|c|c|c|c|c|c|c|}
\hline \multicolumn{2}{|c|}{ EOATNMR } & Média & Mediana & Desvio Padrão & QI & Q3 & $N$ & IC & p-valor \\
\hline $\begin{array}{l}\text { OD } \\
(\mathrm{I}, 5 \mathrm{kHz})\end{array}$ & $\begin{array}{l}\text { ComRGE } \\
\text { SemRGE }\end{array}$ & $\begin{array}{l}7,95 \\
9,05\end{array}$ & $\begin{array}{l}9 \\
9\end{array}$ & $\begin{array}{l}8,21 \\
6,38\end{array}$ & $\begin{array}{c}3 \\
6,5\end{array}$ & $\begin{array}{l}12 \\
14\end{array}$ & $\begin{array}{l}63 \\
55\end{array}$ & $\begin{array}{l}2,03 \\
1,69\end{array}$ & 0,374 \\
\hline $\begin{array}{l}\mathrm{OD} \\
(2 \mathrm{kHz})\end{array}$ & $\begin{array}{l}\text { ComRGE } \\
\text { SemRGE }\end{array}$ & $\begin{array}{l}3,73 \\
7,71\end{array}$ & $\begin{array}{l}5 \\
8\end{array}$ & $\begin{array}{l}9,38 \\
5,65\end{array}$ & $\begin{array}{c}-1 \\
5\end{array}$ & $\begin{array}{c}8 \\
10,5\end{array}$ & $\begin{array}{l}63 \\
55\end{array}$ & $\begin{array}{l}2,32 \\
1,49\end{array}$ & $0,00 \mid *$ \\
\hline $\begin{array}{l}\text { OD } \\
(2,5 \mathrm{kHz})\end{array}$ & $\begin{array}{l}\text { ComRGE } \\
\text { SemRGE }\end{array}$ & $\begin{array}{r}-1,48 \\
5,87\end{array}$ & $\begin{array}{l}-1 \\
7\end{array}$ & $\begin{array}{l}9,92 \\
7,53\end{array}$ & $\begin{array}{l}-6 \\
2\end{array}$ & $\begin{array}{l}3,5 \\
10,5\end{array}$ & $\begin{array}{l}63 \\
55\end{array}$ & $\begin{array}{l}2,45 \\
1,99\end{array}$ & $<0,\left.00\right|^{*}$ \\
\hline $\begin{array}{l}\text { OD } \\
(3 \mathrm{kHz})\end{array}$ & $\begin{array}{l}\text { ComRGE } \\
\text { SemRGE }\end{array}$ & $\begin{array}{r}-4,38 \\
4,56\end{array}$ & $\begin{array}{l}-2 \\
7\end{array}$ & $\begin{array}{l}9,72 \\
8,41\end{array}$ & $\begin{array}{l}-9 \\
-2\end{array}$ & $\begin{array}{l}1 \\
9\end{array}$ & $\begin{array}{l}63 \\
55\end{array}$ & $\begin{array}{r}2,4 \\
2,22\end{array}$ & $<0,00 \mid *$ \\
\hline $\begin{array}{l}\mathrm{OD} \\
(3,5 \mathrm{kHz})\end{array}$ & $\begin{array}{l}\text { ComRGE } \\
\text { SemRGE }\end{array}$ & $\begin{array}{l}-3,4 \\
5,29\end{array}$ & $\begin{array}{c}-1 \\
6\end{array}$ & $\begin{array}{l}9,69 \\
8,38\end{array}$ & $\begin{array}{l}-9,5 \\
0,5\end{array}$ & $\begin{array}{c}4 \\
10\end{array}$ & $\begin{array}{l}63 \\
55\end{array}$ & $\begin{array}{r}2,39 \\
2,21\end{array}$ & $<0,00$ I* \\
\hline $\begin{array}{l}\mathrm{OD} \\
(4,5 \mathrm{kHz})\end{array}$ & $\begin{array}{l}\text { ComRGE } \\
\text { SemRGE }\end{array}$ & $\begin{array}{c}-3,87 \\
7\end{array}$ & $\begin{array}{l}-2 \\
7\end{array}$ & $\begin{array}{l}9,61 \\
8,89\end{array}$ & $\begin{array}{c}-10 \\
3\end{array}$ & $\begin{array}{l}3,5 \\
12\end{array}$ & $\begin{array}{l}63 \\
55\end{array}$ & $\begin{array}{r}2,37 \\
2,35\end{array}$ & $<0,\left.00\right|^{*}$ \\
\hline $\begin{array}{l}\text { OE } \\
(\mathrm{I}, 5 \mathrm{kHz})\end{array}$ & $\begin{array}{l}\text { ComRGE } \\
\text { SemRGE }\end{array}$ & $\begin{array}{c}7,1 \\
7,22\end{array}$ & $\begin{array}{l}8 \\
8\end{array}$ & $\begin{array}{l}8,44 \\
5,77\end{array}$ & $\begin{array}{l}2 \\
4\end{array}$ & $\begin{array}{l}12 \\
11\end{array}$ & $\begin{array}{l}63 \\
55\end{array}$ & $\begin{array}{l}2,09 \\
1,52\end{array}$ & 0,827 \\
\hline $\begin{array}{l}\text { OE } \\
(2 \mathrm{kHz})\end{array}$ & $\begin{array}{l}\text { ComRGE } \\
\text { SemRGE }\end{array}$ & $\begin{array}{l}3,05 \\
6,42\end{array}$ & $\begin{array}{l}3 \\
7\end{array}$ & $\begin{array}{l}8,79 \\
5,68\end{array}$ & $\begin{array}{c}-1 \\
3\end{array}$ & $\begin{array}{l}8,5 \\
10\end{array}$ & $\begin{array}{l}63 \\
55\end{array}$ & $\begin{array}{l}2,17 \\
1,5\end{array}$ & $0,019 *$ \\
\hline $\begin{array}{l}\text { OE } \\
(2,5 \mathrm{kHz})\end{array}$ & $\begin{array}{l}\text { ComRGE } \\
\text { SemRGE }\end{array}$ & $\begin{array}{r}-3,19 \\
4,44\end{array}$ & $\begin{array}{l}0 \\
6\end{array}$ & $\begin{array}{l}11,71 \\
7,18\end{array}$ & $\begin{array}{l}-6 \\
0\end{array}$ & $\begin{array}{l}3 \\
9\end{array}$ & $\begin{array}{l}63 \\
55\end{array}$ & $\begin{array}{l}2,89 \\
1,9\end{array}$ & $<0,\left.00\right|^{*}$ \\
\hline $\begin{array}{l}\text { OE } \\
(3 \mathrm{kHz})\end{array}$ & $\begin{array}{l}\text { ComRGE } \\
\text { SemRGE }\end{array}$ & $\begin{array}{r}-5,68 \\
3,82\end{array}$ & $\begin{array}{l}-5 \\
5\end{array}$ & $\begin{array}{l}9,19 \\
9,07\end{array}$ & $\begin{array}{l}-9 \\
-2,5\end{array}$ & $\begin{array}{l}-0,5 \\
10,5\end{array}$ & $\begin{array}{l}63 \\
55\end{array}$ & $\begin{array}{l}2,27 \\
2,4\end{array}$ & $<0,\left.00\right|^{*}$ \\
\hline $\begin{array}{l}\text { OE } \\
(3,5 \mathrm{kHz})\end{array}$ & $\begin{array}{l}\text { ComRGE } \\
\text { SemRGE }\end{array}$ & $\begin{array}{l}-5,02 \\
4,95\end{array}$ & $\begin{array}{l}-4 \\
5\end{array}$ & $\begin{array}{l}8,54 \\
8,18\end{array}$ & $\begin{array}{c}-9,5 \\
\mid\end{array}$ & $\begin{array}{l}1,5 \\
9\end{array}$ & $\begin{array}{l}63 \\
55\end{array}$ & $\begin{array}{l}2,11 \\
2,16\end{array}$ & $<0,\left.00\right|^{*}$ \\
\hline $\begin{array}{l}\text { OE } \\
(4,5 \mathrm{kHz})\end{array}$ & $\begin{array}{l}\text { ComRGE } \\
\text { SemRGE }\end{array}$ & $\begin{array}{l}-5,46 \\
5,51\end{array}$ & $\begin{array}{c}-4 \\
8\end{array}$ & $\begin{array}{l}9,35 \\
9,39\end{array}$ & $\begin{array}{c}-11,5 \\
-4\end{array}$ & $\begin{array}{c}1 \\
\mid 1,5\end{array}$ & $\begin{array}{l}63 \\
55\end{array}$ & $\begin{array}{l}2,31 \\
2,48\end{array}$ & $<0,\left.00\right|^{*}$ \\
\hline
\end{tabular}

Legenda: *p-valor<0,05. Legenda: EOAT; NMR: Nível médio de respostas das emissões otoacústicas por estímulo transientes; Com RGE: com refluxo gastroesofágico; Sem RGE: sem refluxo gastroesofágico.

refluxo, as relações sinal/ ruído obtidas nas EOAT foram estatisticamente significantes maiores do que as obtidas no grupo com refluxo. A maior média da relação sinal/ruído encontrada foi na banda de frequência de $4 \mathrm{kHz}$ em ambas as orelhas, com valores médios de $14,55 \mathrm{~dB} \pm 2,08$ na OD e $13,02 \mathrm{~dB} \pm 2,26$ na OE.

Os resultados dos níveis médios de respostas das EOAT nas bandas de frequência estudadas em ambas as orelhas, aparecem descritos na Tabela 3. Nesta análise foi utilizado o teste de Mann-Whitney.

Foi possível observar que os níveis de respostas médios das EOAT foram maiores no grupo sem refluxo para as bandas de frequências de $2 \mathrm{kHz}, 2,5 \mathrm{kHz}, 3 \mathrm{kHz}$, $3,5 \mathrm{kHz}$ e $4,5 \mathrm{kHz}$ bilateralmente, com diferença estatisticamente significante. Somente na frequência de $1,5 \mathrm{kHz}$, de ambas as orelhas, não houve diferença estatisticamente significante entre os grupos. Os maiores níveis médios de resposta das EOAT foram de $7,71 \mathrm{~dB}$ e $7 \mathrm{~dB}$ encontradas na banda de frequência de $2 \mathrm{e} 4 \mathrm{kHz}$, respectivamente, na OD do grupo sem refluxo.

\section{DISCUSSÃO}

$\mathrm{Na}$ análise dos resultados das EOAT nos grupos estudados (Gráficos 1 e 2), observou-se maior ocorrência de respostas presentes no grupo sem refluxo, contudo observou-se também alta prevalência de respostas presentes no grupo com refluxo, nas bandas de frequências de 1,5; 3 e 3,5kHz. Este achado discorda do estudo que observou a presença das EOA apenas nas frequências de 2 e $4 \mathrm{kHz}$ em crianças com otite média secretora (13).

$\mathrm{Na}$ análise da ocorrência das EOAT com o uso de medicação em lactentes do grupo com refluxo (Tabela 1), observou-se que a maioria dos lactentes que estavam em uso da medicação falhou mais nas EOAT em comparação aos lactentes que nunca usaram a medicação, com diferença estatisticamente significante. Como o RGE pode causar alteração de orelha média $(9,10)$ e esta por sua vez, interfere na captação das EOAT, é possível que a ausência de repostas no grupo que usava medicação, tenha ocorrido devido ao período mais crítico do RGE, ou seja, vômitos exagerados, 
que por sua vez, podem causar doença da tuba auditiva e propiciar alterações de orelha média $(10,12,14,15,16)$.

Estudos relataram que o tratamento do RGE melhora os sintomas otorrinolaringológicos $(10,17,18)$. Isso pode justificar a alta prevalência de EOAT presentes nos lactentes que já haviam feito uso de medicação, comparado ao que ainda estavam em uso. As EOA estão presentes em orelha funcionalmente normais e deixam de ser captadas quando apresentam limiares acima de 30dBNA ou alteração de orelha média. $(1,2,4,6)$

Quando foi analisado os níveis de relação sinal por banda de frequência (Tabela 2) comparando-se os grupos, observou-se diferença estatisticamente significante em todas as bandas de frequência: O grupo com refluxo apresentou menores níveis de relação sinal ruído.

Foi possível ainda observar que os maiores níveis de relação sinal ruído das EOAT ocorreram nas bandas de frequência de 3 e $4 \mathrm{kHz}$, no grupo sem refluxo, em ambas as orelhas e nas bandas de 3 e $3,5 \mathrm{kHz}$ no grupo com refluxo. Um estudo realizado com 100 recém nascidos encontrou EOA espontâneas presentes, com maior concentração nas bandas de frequência de 3 e $4 \mathrm{kHz}$ bilateralmente, em RN a termo e sem indicadores de risco. Isso pode justificar uma maior relação sinal/ruído nestas bandas de frequência. Outro estudo relata o registro das EOA em recém nascidos é sempre maior nas bandas de frequência aguda, devido à anatomia da orelha, sendo importante na detecção de perdas auditivas $(3,4,6,19)$.

A relação sinal/ruído é uma das análises avaliadas no critério de passa/falha das EOAT e para que esta seja considerada como passa, faz-se necessário à integridade de orelha média para melhor captação dessa relação, evitando resultados falsos positivos (20).

Comparando os níveis de resposta das EOAT (Tabela 3), foi possível observar que o grupo sem refluxo apresentou maiores respostas nas bandas de frequência de $2 \mathrm{a} 4 \mathrm{kHz}$, com diferença significativa quando comparada ao grupo com refluxo. Estes achados corroboram com estudos que encontraram maior amplitude das EOAT nas frequências altas e redução da amplitude nas frequências baixas $(21,22)$, podendo ser atribuído, ao aumento da amplitude nestas frequências, as EOA espontâneas que ocorreram entre 3 e $4 \mathrm{kHz}$ (23).

Estudos relataram que a orelha média de neonatos é dominada por massa e por baixa frequência de ressonância (24) e que a frequência de ressonância de orelhas com efusão ocorre em $858 \mathrm{~Hz}$ com desvio padrão de $483 \mathrm{~Hz}$ (25) e que a alteração de orelha média afeta a amplitude das EOA (26).
Analisando os grupos separadamente, foi possível ainda observar que em ambos ocorreram uma maior prevalência de EOAT presentes. Um dado que chama a atenção é que mesmo com EOAT presente observou-se um diminuição da amplitude nas EOAT do grupo com refluxo gastroesofágico quando comparado ao grupo sem refluxo. Estudos $(7,8,25,26)$ afirmaram que o RGE pode contribuir para inflamação de orelha média e que esta resulta na redução significativa do nível de resposta das EOAT.

\section{CONCLUSÃO}

Houve diminuição significativa da ocorrência e dos níveis de resposta das emissões otoacústicas evocadas por estímulo transiente em lactentes com refluxo gastroesofágico fisiológico quando comparados a crianças sem refluxo.

\section{REFERÊNCIAS BIBLIOGRÁFICAS}

1. Kemp DT. Otoacoustic emissions, their origin in cochlear function, and use. British Medical Bulletin. 2002, 63:223241.

2. Kemp DT, Bray P, Alexander L, Brown AM. Acoustic emission cochleography; pratical aspects. Scand. Audiol. Suppl. 1986, 25:71-85.

3. Gattaz G, Cerruti VQ. O uso do registro de emissões otoacústicas evocadas para triagem auditiva em neonatos de risco para deficiência auditiva. Revista Paulista de Pediatria. 1994, 12(3):291-4.

4. Pialarissi PR, Gattaz G. Emissões otoacústicas: conceitos básicos e aplicações clínicas. Rev Arquivos da Fundação Otorrinolaringologia. 1997, 1(2):13-6.

5. DellAringa Alfredo R, DellAringa Ana Helena Bannwart, Juares Antônio JC, Melo Cinthia de, Perches Filho Renato M.Emissões otoacústicas por produtos de distorção em crianças de 2 a 7 anos. Rev. Bras. Otorrinolaringol. [serialontheInternet]. 2004 June [cited 2010 Oct 18], 70(3):380384. Availablerom: http://www.scielo.br/ scielo.php?script=sci_arttext\&pid=S0034$72992004000300014 \& \operatorname{lng}=$ en.doi:10.1590/S003472992004000300014 .

6. Choi SS, Pafitis IA, Zaizal GH, Paterl KM. Clinical Applications of transienty evoked otoacustic emissions in the pediatric population. Ann Otol Rhinol Laryngol. 1999, 108:132-8.

7. Tasker A, Dettmar PW, Panetti M, Koufman JÁ, Birchall JP, Pearson JP. Is gastric reflux a cause of otitis media with 
effusion in children? Laryngoscope. 2002, 112(11):19301934

8. Crapko M, Kerschner JE, Syring M, Johnston N. Role of extra-esophageal reflux in chronic otitis media with effusion. Laringoscope. 2007, 117:1419-23.

9. Carré IJ.The natural history of partial thoracic stomach (hiatus hernia) in children. Arch Dis Child. 1959, 34:34453.

10. Shepherd RW, Wren J, Evans S, Ong TH, Lander M. Gastroesophageal reflux in children; clinical profile course and outcome with active therapy in cases. Clin Pediatr. 1987, 26:55-60

11. Roma Foundation.org [home page the internet]. McLean, VA: Roma Foundation Introduces Diagnostic Algorithms for Common Gastrointestinal Symptoms, Inc.; c2009 [updated 2009 Fev 10; cited 2009 Mar 9]. Avaliable from: http:// www.romecriteria.org > acesso em: 18 agosto de 2010 às 21:00 horas.

12. Pediatric Gastroesohpageal Reflux Clinical Practice Guidelines were published in the Journal of Pediatric Gastroenterology and Nutrition 2001; Volume 32: Supplement 2 pages 1-31. Complete or: www.cdhnf.org or www.naspghan.org > acesso em: 05 julho de 2010 às 23:34 horas.

13. Van Cauwenberge PB, Vinck BM, De Vel E, Dhooge I. Tympanometry and click evoked otoacoustic emissions in secretory otitis media: Are c-eoae really consistently absent in type b tympanograms. Sixth international symposium on recent Advance in otitis media. 1995 June; Ft. Lauderdale, FL.

14. Quintella T. Refluxo gastroesofágico e doença otorrinolaringológica na infância. In: Sih T e col. Otorrinolaringologia Pediátrica. Rio de janeiro: Revinter, 1998. p.17-21.

15. Velepic M, Rozmanic V, Velepic M, Bonifacie M. Gastroesophageal reflux, allergy and chronic tubotympanal disorders in children. Int J Pediatr Otorhinolaryngol. 2000, 55:187-90.
16. Crapko M, Kerschner JE, Syring M, Johnston N. Role of extra-esophageal reflux in chronic otitis media with Effusion. Laringoscope. 2007, 117:1419-23.

17. Halstead LA. Role of gastroesophageal reflux in pediatric upper airway disorders. Otolaringol Head Neck Surg. 1999, 120:208-14.

18. Megale SRMCL, Scanavini ABA, Andrade EC, Fernandes MIM, Anselmo-Lima WT. Gastroesophageal reflux disease: Its importance in ear, nose, and throat practice. Int J Pediatr Otorhinolaryngol. 2006, 70:81-8.

19. Speri MRB, Pratesi R. Emissões otoacústicas transients e espontâneas em recém nascidos a termo. Artigos distúrbio da comunicação. São Paulo. 2004, 16(1):9-16.

20. Finitzo T, Albright K, Oneal J. The newborn with hearing loss: detection in the nursey. Pediatrics. 1998, 102(6):145260.

21. Basseto M, Chiari BM, Azevedo MF. Emissões otoacústicas evocadas transientes (EOAT): amplitude da resposta em recém-nascidos a termo e pré-termo. Rev Bras Otorrinolaringol. 2003, 69(1):84-92.

22. Naeve SL, Margolis RH, Levine SC, Fournier EM. Effects of ear-canal air pressure on evoked otoacoustic emissions. J Acoustic Soc Am. 1992, 91:2091-5.

23. Holte L, Margolis RH, Cavanaugh RM. Developmental changes in multifrequency tympanograms. Audiology. 1991, 30:1- 24 .

24. MVlachou S, Ferekidis E, Douniadakis D, Apostolopoulos $\mathrm{N}$, Adamopoulos G. Multiple-frequency tympanometry in children with acute otitis media. Otolaryngology - Head Neck Surgery. 1999, 121(6):797-801.

25. Koivunem P, Uhari M, Laitakari K, Alho OP, Luotonem J. Otoacustic emissions and tympanometry in children with otitis media. Ear Hear. 2000, 21;212-17.

26. Azevedo MF. Emissões otoacústicas. Figueredo MS. Emissões otoacústicas e BERA. São José dos Campos: Pulso; 2003. p.53-7 